\title{
Los trastornos bipolares hoy: más allá del DSM-5
}

\author{
Gustavo H. Vázquez ${ }^{1}$
}

\section{Artículo}

Material original autorizado para la publicación en la revista Psicodebate. Facultad de Ciencias Sociales. Universidad de Palermo.

Recibido 28-08-2014 | Aceptado 02-09-2014

\section{Resumen}

Los trastornos bipolares constituyen un complejo grupo de enfermedades mentales recurrentes que presentan una alta prevalencia en la población general. Las clasificaciones actuales, como el ICD-10 y el DSM-5, constituyen un avance significativo para su correcta identificación. Sin embargo, aún existe una prolongada latencia en su diagnóstico correcto. En el presente trabajo se describen las características clínicas más distintivas de estas enfermedades, y se discuten los conceptos generales del espectro bipolar, los estados mixtos, las diferencias entre la depresión unipolar y bipolar, y los criterios clínicos diferenciales con otros trastornos mentales.

Palabras Clave: Trastornos Bipolares - DSM-5 - Espectro Bipolar - Estados mixtos - Depresión Bipolar. .

1 Centro de Investigaciones en Neurociencias y Neuropsicología. Universidad de Palermo, Buenos Aires, Argentina. International Consortium for Bipolar Disorder Research. McLean Hospital Division of Massachusetts General Hospital. Harvard University, Boston, EEUU; gvazquez@palermo.edu 
Vázquez I Psicodebate, 14(2) I 9-24.

\section{Abstract}

Bipolar disorders constitute a complex group of recurrent mental illnesses with high prevalence among general population. The current systems of classification, such as CIE-10 and DSM-5, represent a significant advance for their accurate identification. However, there is still a relevant delay on their diagnoses. The present paper describes the more distinctive clinical features of these illnesses, discuss the general concepts regarding of bipolar spectrum, mixed states, clinical differences between unipolar and bipolar depression, and the differential clinical criteria with other disorders.

Keywords: Bipolar Disorders - DSM-5 - Bipolar Spectrum - Mixed States Bipolar Depression. 
Los manuales oficiales de diagnóstico en psiquiatría han denominado como trastorno bipolar a la entidad clínica que había sido conocida durante la mayor parte del siglo pasado como psicosis maníaco depresiva. La definición actual del trastorno bipolar brindada por el DSM-5 y el ICD-10 (American Psychiatric Association, 2013; Organización Mundial de la Salud, 1992) es bastante más estrecha que el concepto original de la entidad descripta por Kraepelin (1996). La importancia de la detección de un episodio maníaco agudo, generalmente con síntomas psicóticos, en el curso de una enfermedad con episodios depresivos, es fácilmente compartida por los especialistas como la piedra fundamental para el diagnóstico del trastorno bipolar I. Aunque también la hipomanía es reconocida como un cuadro determinante para el diagnóstico de bipolaridad (en este caso trastorno bipolar II), algunos investigadores consideran que el umbral diagnóstico para esta entidad particular es demasiado alto y que además tendría excesivos criterios de exclusión. Por tal motivo, las publicaciones sobre trastornos afectivos que se generan mediante la utilización de instrumentos o a partir de investigaciones con los criterios conservativos estiman que la bipolaridad está presente en el 1\% de la población, y que sólo representa del 10 al 15\% de todos los trastornos del ánimo. En una encuesta norteamericana a nivel nacional se ha reportado una tasa de prevalencia a lo largo de la vida para el trastorno bipolar de 2,1\% (Merikangas et al., 2007). Por otro lado, la edad de inicio del trastorno bipolar ha sido calculada en alrededor de los 25 años en varios países del mundo (Baldessarini et al., 2012a; Perlis et al., 2004; Vázquez Lolich, Leiderman, Tondo, \& Baldessarini, 2012).

El trastorno bipolar es habitualmente subdiagnosticado, en general como depresión unipolar, y conlleva una importante demora diagnóstica. En una investigación llevada a cabo en los Estados Unidos por la Depressive and ManicDepressive Illness National Association sobre una gran población de pacientes bipolares, el 48\% había sido diagnosticado como bipolar sólo luego de haber visto a tres o más psiquiatras, el $57 \%$ había recibido un diagnóstico incorrecto (44\% depresión unipolar y 34\% esquizofrenia) (Lish, Dime-Meenan, \& Whybrow, 1994). En un estudio colaborativo internacional multicéntrico se replican las tasas de retraso diagnóstico en países muy distintos geográfica y socio-culturalmente (Baldessarini et al, 2012a). Además, tanto en nuestra región como en nuestro país, contamos también con estudios relativamente recientes que arrojan cifras igualmente alarmantes (Strejilevich \& Retamal Carrasco, 2003; Lolich, Vázquez, $\&$ Leiderman, 2010). Estos estudios muestran que el promedio en que el paciente había recibido el diagnóstico correcto fue de 8 a 10 años, con la consecuente demora tanto en recibir un tratamiento adecuado como, incluso, en haber evitado recibir medicación inapropiada y potencialmente nociva. Este último punto en cuanto al diagnóstico precoz es bastante crítico, ya que el suicidio en los pacientes 
bipolares suele ocurrir durante la primera década de la enfermedad (Nordentoft, Mortensen, \& Pedersen, 2011).

A su vez, el diagnóstico equivocado y, como consecuencia, el tratamiento inadecuado de la depresión bipolar con antidepresivos en monoterapia y sin estabilizantes del ánimo, puede llevar a un aumento en el riesgo de switch o viraje (que es cuando el paciente con trastorno bipolar pasa de una fase anímica a la opuesta) hacia la manía o hipomanía (Tondo, Vázquez, \& Baldessarini, 2010), y en la aparición de ciclado rápido (el paciente presenta cuatro o más episodios afectivos en el lapso de un año) (Kupka et al., 2005).

\section{Criterios diagnósticos actuales}

La Asociación Americana de Psiquiatría, a través del DSM-5 publicado en mayo del 2013, establece criterios clínicos bien definidos para el diagnóstico de los episodios de manía e hipomanía, que constituyen los cuadros sintomatológicos distintivos para el trastorno bipolar (American Psychiatric Association, 2013). Se considera que tanto la hipomanía como la manía comparten los mismos síntomas cardinales de ánimo anormalmente elevado, expansivo o irritable, acompañado del aumento persistente de la actividad o energía, a los que se suman tres o más síntomas diversos, tales como autoestima exagerada o grandiosidad, disminución de la necesidad de dormir, verborrea, fuga de ideas o experiencia subjetiva de que el pensamiento está acelerado, distraibilidad, agitación psicomotora, e implicación excesiva en actividades placenteras que tienen un alto potencial para producir consecuencias graves. Sin embargo, ambos sindromes se diferencian en su duración (al menos una semana para manía y de al menos 4 días para hipomanía) y en el impacto funcional que producen en el individuo, ya que sólo la manía llega a provocar un deterioro significativo en lo laboral, en las actividades o en las relaciones sociales. Por otro lado, la necesidad de hospitalización o la presencia de síntomas psicóticos son exclusivas del episodio maníaco (American Psychiatric Association, 2013).

Los episodios maníacos no siempre se presentan con la sintomatología eufórica clásica, sino que en realidad la manía tiene formas clínicas distintas, descriptas desde hace más de un siglo atrás por Emil Kraepelin (1996). En un estudio reciente, y a partir de las manifestaciones clínicas de más de 100 pacientes maníacos, Disalver, Chen, Shoaib, y Swann (1999) establecieron tres variantes distintivas: la manía eufórica (clásica o pura), la manía disfórica (con irritabilidad y agresividad) y la manía depresiva (con presencia de síntomas severos del polo opuesto).

Las formas clínicas del trastorno bipolar estipuladas por el DSM-5 (tabla 1) incluyen el trastorno bipolar tipo I, determinado por la aparición de un episodio 
maníaco, con o sin episodios previos de depresión mayor; el trastorno bipolar tipo II, caracterizado por la presencia de un cuadro hipomaníaco en un paciente con antecedentes de episodios depresivos mayores; y el trastorno ciclotímico donde se exige la presencia, a lo largo de por lo menos dos años, de períodos de síntomas hipomaníacos y depresivos que no cumplen los criterios de duración para un episodio afectivo mayor. El diagnóstico de otros trastornos bipolares especificados queda relegado para aquellas entidades clínicas ("atípicas") del trastorno bipolar que no cumplen con los criterios para los cuadros anteriores (American Psychiatric Association, 2013). En general, son pacientes con episodios depresivos mayores que presentan algunos rasgos de bipolaridad, como historia familiar de bipolaridad, síntomas hipomaníacos que duran menos de 4 días, episodios hipomaníacos sin antecedentes de episodios depresivos mayores, trastornos ciclotímicos de menos de dos años de evolución, o manías o hipomanías inducidas por fármacos. En esta última versión del DSM, los virajes anímicos secundarios a la acción de los antidepresivos son un criterio para el diagnóstico de trastorno bipolar. Es importante tener presente que el paciente que sufre un trastorno bipolar I, además de la manía, puede presentar también episodios hipomaníacos durante su evolución.

\section{Tabla 1.}

Las cuatro formas clínicas del trastorno bipolar (según el DSM-5).

1 Trastorno Bipolar I: episodio de manía, con o sin depresión mayor

2 Trastorno Bipolar II: hipomanía, con depresión mayor

3 Trastorno ciclotímico: hipomanía+síntomas depresivos leves $>2$ años

4 Otros Trastornos Bipolares Especificados: cuadros con "rasgos" de bipolaridad

\section{El concepto de espectro afectivo-bipolar}

El concepto de espectro afectivo intenta reflejar la idea de que los trastornos del ánimo tendrían raíces hereditarias y/o biológicas y/o de desarrollo temprano similares, pero se manifestarían fenotípicamente como enfermedades unipolares y bipolares (Angst, 1998). A partir de este constructo teórico inicial y en el campo de la bipolaridad, surge el llamado espectro bipolar, que es un término más amplio aplicado a los trastornos bipolares, y, más allá de la manía clásica (o bipolar I), modifica los conceptos actuales de trastorno bipolar II ya que incluye, según Akiskal y Vazquez (2006), el trastorno esquizobipolar, la manía, los estados mixtos, las depresiones con hipomanías (indistintas de su duración), las hipomanías inducidas farmacológicamente por antidepresivos o drogas de 
abuso (o bipolar III), depresiones instaladas sobre temperamentos ciclotímicos e hipertímicos (bipolar IV), depresiones recurrentes con historia familiar de bipolaridad o respondedoras al litio (y por extensión a los demás estabilizantes del ánimo) (bipolar V), y los episodios hipomaníacos instaurados en cuadros con deterioro cognitivo (por ejemplo, enfermedad de Alzheimer) inicial moderado a severo (bipolar VI) (tabla 2).

Tabla 2.

El espectro bipolar

(Akiskal, Cetkovich-Bakmas, García-Bonetto, Strejilevich, \& Vázquez, 2006).

\begin{tabular}{ll}
\hline Subtipo bipolar & Características clínicas distintivas \\
\hline T Bip $1 / 2$ & Trastorno esquizoafectivo tipo bipolar \\
T Bip I & Al menos un episodio de manía o mixto \\
T Bip I 1/2 & Depresiones con hipomanías prolongadas \\
T Bip II & Depresiones con hipomanía \\
T Bip II 1/2 & Depresiones con inestabilidad anímica \\
T Bip III & Hipomanía por antidepresivos \\
T Bip III 1/2 & Cambios anímicos por abuso de sustancias / alcohol \\
T Bip IV & Depresiones sobre temperamentos hipertímicos (o ciclotímicos) \\
T Bip V & Depresiones cíclicas recurrentes (>5) con síntomas hipomaníacos, \\
& antecedentes familiares de bipolaridad y respuesta + a estabilizantes \\
T Bip VI & Inestabilidad anímica y/o hipomanías en pacientes con demencia \\
& temprana \\
\hline
\end{tabular}

\section{Evolución clínica y diagnósticos diferenciales}

El trastorno bipolar suele manifestarse de manera precoz, a edades tempranas, en forma de cuadros depresivos de inicio brusco, con intervalo de remisión posterior, que tienden a repetirse en el tiempo, independizándose gradualmente de los detonantes o estresores externos, volviéndose cada vez más intensos y prolongados, con períodos de sintomatología subsindromática leve entre cada uno de los episodios (Baldessarini et al., 2012b; Goodwin \& Jamison, 2007). Esta descripción general corresponde al mayor porcentaje de pacientes bipolares. Sin embargo no debe perderse de vista que el inicio y el curso de la enfermedad puede ser tan variado y diferente como los múltiples subtipos clínicos.

La frecuente demora en el diagnóstico temprano de la enfermedad puede ser atribuida, en el caso de la depresión bipolar, a la ausencia de reporte de fases previas hipomaníacas por parte del paciente o la familia, y a la falta de 
reconocimiento de las mismas por parte de los profesionales, que llevan al diagnóstico erróneo de depresión unipolar (Angst et al., 2012).

En el caso de los cuadros maníacos agudos, la excitación psicomotriz y la sintomatología delirante y/o alucinatoria, que habitualmente no se considera como propia de los pacientes afectivos, puede llevar al diagnóstico equivocado de un trastorno esquizofrénico (Akiskal \& Vazquez, 2006).

A su vez, el error diagnóstico lleva al equipo de salud a instaurar un tratamiento equivocado, que en el caso de la depresión bipolar, podría conducir al agravamiento de la enfermedad. Se ha demostrado que el tratamiento de la depresión en monoterapia con antidepresivos en el contexto del trastorno bipolar produce una mayor tasa de switch o viraje hacia la euforia y el aumento del número de ciclos afectivos (Tondo et al., 2010). A su vez, el tratamiento con fármacos antipsicóticos clásicos o neurolépticos a largo plazo de un cuadro maníaco, induce la aparición de episodios depresivos prolongados, que suelen ser interpretados como síntomas negativos propios de los pacientes con esquizofrenia (Vieta et al., 2009).

Se ha calculado que un paciente bipolar presenta alrededor de diez episodios afectivos a lo largo de su vida (Goodwin \& Jamison, 2007). Los pacientes bipolares tipo II suelen tener más cantidad de episodios depresivos y padecer un mayor número de episodios afectivos que los bipolares tipo I (Goodwin \& Jamison, 2007). El riesgo de suicidio suele ser más alto entre los pacientes depresivos bipolares II que entre los unipolares y los bipolares I (Nordentoft et al., 2011).

La ciclación rápida está definida como el padecimiento de cuatro o más episodios afectivos de cualquier tipo (manía, hipomanía, o depresión) en el transcurso de un año (American Psychiatric Association, 2013). Este tipo de curso en el trastorno bipolar puede presentarse en cualquier momento evolutivo de la enfermedad, y es más frecuente entre las mujeres y entre los pacientes bipolares tipo II. Todas estas características clínicas a menudo pueden inducir al diagnóstico de un trastorno de personalidad limitrofe o borderline, sobre todo si existe una intensa labilidad y oscilación afectiva (Akiskal \& Vázquez, 2006).

\section{La depresión en el contexto del trastorno bipolar}

El diagnóstico de depresión bipolar tipo II en particular es fundamental por las implicancias en el pronóstico que conlleva como entidad propia. Las depresiones bipolares tipo II suelen tener un inicio más temprano en la vida de los pacientes que las unipolares, tienden a cronificarse, poseen características clínicas de atipicidad y tienen menos períodos de eutimia (Baldessarini et al., 2012b). Además, y a pesar de que los síntomas depresivos en los bipolares II pueden ser moderados, estos individuos tienen un bajo nivel de funcionamiento (Rosa et al., 
2010), quizás relacionado con la alta frecuencia de cuadros psicopatológicos no afectivos coexistentes (o comórbidos), como los ataques de pánico, las fobias, el abuso de alcohol y de otras drogas, los déficits atencionales y de hiperactividad, los desórdenes alimenticios y los trastornos de personalidad, entre otros (Vázquez, Baldessarini, \& Tondo, 2014; Vieta \& Suppes, 2008).

Uno de los principales factores que llevan al diagnóstico equivocado de depresión unipolar es la ausencia de una toma adecuada de conciencia de los síntomas de euforia por parte del paciente bipolar (Perlis, 2005). Como durante los episodios depresivos el individuo tiene una mayor conciencia de sus síntomas afectivos, la evaluación diagnóstica basada solamente en la entrevista con el paciente puede llevar a un subdiagnóstico de la manía y/o hipomanía, por lo que se recomienda, siempre que sea posible, entrevistar también a un familiar directo o cuidador del paciente depresivo (Goodwin \& Jamison, 2007). En este sentido, en un estudio de Keitner, Solomon y Ryan (1996) sobre los síntomas prodrómicos de los episodios afectivos, los familiares reportaban los síntomas maníacos con el doble de frecuencia en comparación con los pacientes ( $47 \%$ contra $22 \%$ ), mientras que los síntomas depresivos habían sido informados con la misma frecuencia tanto por los pacientes como por los familiares.

Los síntomas depresivos suelen causar una mayor disconformidad que los maníacos o hipomaníacos; es por ello que los pacientes acuden habitualmente a consultar al médico durante las fases depresivas y casi nunca durante la hipomanía (Ghaemi, 2003). En general, los pacientes no recuerdan estos episodios de euforia leve y pasajera y, por supuesto, no los consideran como parte de una enfermedad afectiva sino como un período de bienestar (Akiskal, 2005). Sin embargo, y con ayuda de los familiares cercanos, debemos indagar en los enfermos depresivos sobre la aparición, en los últimos días o semanas, de un estado de ánimo elevado, de una actividad desmedida, de una reducción en las horas del sueño, del aumento de energía y proyectos, y de impulsividad y conductas riesgosas, desinhibidas o inapropiadas, que claramente no son parte de la "forma de ser" habitual del paciente (Strejilevich et al., 2010; Vázquez et al., 2005).

\section{Criterio clínico diferencial entre depresión bipolar y depresión unipolar}

Aunque existen muchos libros y trabajos publicados que describen las características clínicas particulares de los cuadros depresivos mayores, muy pocos hacen hincapié en la diferenciación clínica entre los cuadros unipolares y bipolares. En realidad, el diagnóstico de depresión unipolar debe ser un diagnóstico por descarte: primero, por supuesto, tenemos que asegurarnos de que no se trata de una depresión secundaria (es decir, por enfermedad médica 
o por sustancias) y después deberíamos buscar dentro del cuadro depresivo los indicadores de bipolaridad. Lamentablemente, y como lo demuestran numerosas investigaciones, el primer diagnóstico asignado a un síndrome depresivo es el de depresión unipolar primaria (Angst et al., 2012; Dunner, 2003).

En general, se ha instaurado la idea de que la depresión bipolar se caracteriza fundamentalmente por manifestarse como un cuadro de depresión inhibida. En realidad, también se han reportado formas depresivas bipolares con agitación. Es por eso que más allá de la presentación clínica, el diagnóstico diferencial debería basarse además en otros parámetros particulares (Angst et al., 2012). En la tabla 3 se resumen las características distintivas más relevantes que podemos encontrar en la literatura para ambos trastornos.

\section{Tabla 3.}

Características diferenciales entre la depresión bipolar y unipolar.*

\begin{tabular}{lcc}
\hline & Depresión Bipolar & Depresión Unipolar \\
\hline Abuso de sustancias & Muy frecuente & Moderado \\
Historia de hipomanía/manía & Sí & No \\
Temperamento & Ciclotímico & Distímico \\
Distribución por Sexo & Mujeres=Hombres & Mujeres>Hombres \\
Edad de comienzo & Adolescencia, 20 y 30 años & 30 a 50 años \\
Inicio del episodio & Generalmente agudo & Más insidioso \\
Cantidad de Episodios & Numeroso & Menor \\
Duración de los episodios & 3 a 6 meses & 3 a 12 meses \\
Episodios de inicio posparto & Más frecuente & Menos frecuente \\
Episodios psicóticos & Más frecuente & Menos frecuente \\
Actividad psicomotora & Retardada>agitada & Agitada>retardada \\
Sueño & Hipersomnia>insomnio & Insomnio>hipersomnia \\
Historia familiar bipolar & Alta & Baja \\
Historia familiar unipolar & Alta & Alta \\
\hline *Modificado de Akiskal (2005), Baldessarini et al. (2010), y Souery et al. (2012). \\
\hline
\end{tabular}

Es evidente que ante tal cantidad de características diferenciales habría que tener una gran experiencia y conocimiento de los posibles indicadores clínicos de bipolaridad. Es por ello que para facilitar la detección, y en un intento por operacionalizar el diagnóstico de depresión bipolar (es decir, de aquellos cuadros depresivos que en realidad pertenecen al denominado espectro bipolar), Ghaemi (2003) propone una serie de criterios que siguen las directrices principales de la 
tabla 3, y que permiten, más allá de los criterios tradicionales del DSM para los tipos I y II, realizar el diagnóstico de un paciente bipolar en su fase depresiva:

A - Por lo menos un episodio depresivo mayor

B - Sin episodios hipo/maníacos espontáneos

$\mathrm{C}$ - Cualquiera de los siguientes más dos ítems del criterio D o los dos juntos más un ítem del criterio D:

1- Historia familiar de 1er grado de Trastorno Bipolar

2- Inducción de hipo/manía por antidepresivos (ATD)

D - Si no hay ningún ítem del criterio $\mathrm{C}$, seis de los nueve ítems siguientes:

1- Personalidad hipertímica

$2-$ Episodios depresivos recurrentes $(>3)$

3 - Episodios depresivos breves ( $<3$ meses)

4- Síntomas depresivos atípicos (según DSM)

5- Episodios depresivos psicóticos

6 - Inicio depresivo temprano $(<25$ años $)$

7- Depresión postparto

8- Agotamiento de respuesta ATD

9- Falta de respuesta a 3 o más pruebas con ATD

\section{Los estados mixtos}

Los estados mixtos se caracterizan por presentar al mismo tiempo una combinación de síntomas depresivos y maníacos, y desde la psiquiatría clásica han sido considerados como el elemento unificador de la enfermedad maníaco depresiva, sugiriendo que la manía y la depresión son expresiones diferentes de una misma enfermedad. Los criterios diagnósticos del DSM-IV-TR para el episodio mixto exigían la presencia, de manera simultánea, de todos los síntomas necesarios para conformar un episodio depresivo mayor y un episodio maníaco (American Psychiatric Association, 2000). Clínicamente, sin embargo, es excepcional poder encontrar episodios mixtos con los síntomas completos de un episodio depresivo y maníaco al mismo tiempo (Kupfer et al., 2002). En este sentido, la desaparición de la categoría diagnóstica de episodio mixto del DSM-IV-TR y la consecuente introducción del especificador con características mixtas en el DSM-5, constituye un avance en el aspecto psicopatológico (American Psychiatric Association, 2013). Este especificador mixto puede aplicarse tanto a los episodios hipo/maníacos o depresivos del trastorno bipolar tipo I y II como a la depresión unipolar, y consiste en la presencia de los criterios clínicos completos que definen al episodio afectivo mayor más la presencia simultánea de al menos tres síntomas de la fase opuesta. 
En concordancia con esta modificación nosográfica y acompañando a las descripciones clásicas, el concepto clínico de depresión agitada implica la presencia de algunos síntomas depresivos durante la manía, o algunos síntomas maníacos durante un episodio depresivo (Koukoupoulos et al., 2007). Estas características clínicas particulares de la depresión bipolar han sido recientemente confirmadas por los resultados del estudio denominado STEP-BD, donde se reporta la existencia de depresión bipolar "pura" (es decir sin ningún síntoma de fase hipo/maníaca) en sólo el 31\% de los casos (Goldberg et al., 2009). Asimismo, y en sentido prospectivo, varios estudios de seguimiento longitudinales confirman que los síntomas hipomaníacos subsindromáticos son los mejores predictores de conversión de la depresión mayor unipolar al trastorno bipolar (Swann et al., 2012).

La importancia de los cuadros mixtos bipolares se pone de manifiesto si consideramos su elevada frecuencia en la práctica clínica. Dependiendo de la definición que usemos, del 20 al 74\% de los pacientes afectivos padecen episodios bipolares mixtos (Marneros, Deister, \& Rohde, 1990). Es interesante destacar que estas cifras se han mantenido estables en los últimos 110 años. Kraepelin y Weygandt reportaron, utilizando una definición estrecha, un 20\% de estados mixtos entre los pacientes con trastornos afectivos del hospital psiquiátrico de Heidelberg; la cifra se elevó al 60\% al emplearse criterios diagnósticos más amplios para el episodio mixto (Kraepelin, 1996). Estas cifras son prácticamente las mismas que las reportadas en investigaciones más recientes (Goldberg et al., 2009; Goodwin \& Jamison, 2007). Siguiendo el concepto teórico de Kraepelin acerca de la combinación de los síntomas de manía y depresión con los temperamentos ciclotímicos, hipertímicos y distímicos, Akiskal (1992) formuló la existencia de tres formas diferentes de episodios bipolares mixtos. El episodio mixto tipo I es en general psicótico y con incongruencia afectiva y surge de la combinación de un episodio maníaco en un paciente con temperamento depresivo. El episodio mixto tipo II no es psicótico y es producido por "tormentas ciclotímicas" dentro de un episodio depresivo mayor; este cuadro sería el resultado de un episodio depresivo instaurado sobre un temperamento ciclotímico. El tipo III se origina a partir de un temperamento hipertímico sobre el que se instaura un episodio depresivo mayor y en general se presenta con agitación, hipersexualidad y taquilalia. Es importante destacar que los episodios mixtos pueden presentar síntomas psicóticos, y conllevan un alto riesgo suicida y un curso de tipo crónico.

En todos estos casos la distinción clínica con un episodio depresivo unipolar es prioritaria, y deberíamos ser más cuidadosos en diagnosticarlos como depresiones agitadas, ya que estos pacientes empeoran de manera significativa con el tratamiento antidepresivo, que les genera mayor ansiedad y agitación, insomnio marcado, y en algunos casos, acrecienta los impulsos suicidas (Akiskal \& Tohen, 
2006). Los estudios más recientes (Balazs, Benazzi, Rihmer, Akiskal, \& Akiskal, 2006) sugieren que serían los episodios mixtos los "responsables" de la tendencia suicida reportada con la utilización de algunos antidepresivos, e incluso en el caso de los niños, podría poner de manifiesto el subdiagnóstico de la enfermedad bipolar en esta población, en virtud de los trabajos que reportan que hasta el 50\% de los preadolescentes que padecen depresión "unipolar" desarrollarán un cuadro de manía o hipomanía si se los sigue en el largo plazo (Geller, Zimmerman, Williams, Bolhofner, \& Craney, 2001).

\section{Conclusiones}

Los trastornos bipolares constituyen un grupo de enfermedades mentales crónicas y recurrentes, muchas veces discapacitantes y que generan un alto impacto a nivel social, académico y familiar. En los últimos años se ha producido un enorme debate con relación a su exacta prevalencia y su correcto diagnóstico entre los pacientes con enfermedad mental. En este sentido, los actuales sistemas nosográficos permiten unificar criterios entre los distintos profesionales de la salud a fin de lograr definir de manera adecuada una entidad clínica que en algunos casos suele confundirse con otras patologías del ánimo, como la depresión unipolar, en otros con los trastornos de la personalidad como el trastorno limítrofe o borderline, o incluso con la esquizofrenia.

Diferentes investigadores sostienen que los actuales criterios clínicos serían demasiado restrictivos, y por lo tanto, dejarían por fuera del diagnóstico correcto y de un adecuado tratamiento a muchos individuos que sufren patologías del ánimo recurrentes. De esta manera se postula que la utilización de un abordaje nosográfico dimensional de la enfermedad anímica podría mejorar la evolución clínica y el pronóstico a largo plazo de muchos pacientes. Por otro lado, la ampliación indiscriminada de los límites diagnósticos para la enfermedad bipolar podría dejar expuestos a esos mismos pacientes, entre otros, al potencial padecimiento del estigma que acompaña a estas enfermedades mentales así como a los posibles efectos secundarios de muchos de los tratamientos farmacológicos con los que contamos en la actualidad. Asimismo, la excesiva amplitud en las definiciones de las entidades clínicas podría demorar las investigaciones básicas que buscan encontrar las bases genéticas y neurobiológicas de las enfermedades del ánimo.

Es evidente que, más allá de los avances en los sistemas diagnósticos actuales, necesitamos mayor cantidad de investigaciones y consensos clínicos para poder realizar un diagnóstico preciso más temprano y el subsiguiente abordaje terapéutico adecuado para poder aliviar el elevado impacto global que suelen generar los trastornos bipolares. 


\section{Referencias}

Akiskal, H. S. (1992). The distinctive mixed states of bipolar I, II and III. Clinical Neuropharmacology, 15(1), 632-633.

Akiskal, H. S. (2005). The dark side of bipolarity: detecting bipolar depression in its pleomorphic expressions. Journal of Affective Disorders, 84, 107-115.

Akiskal, H. S. \& Tohen, M. (2006). Bipolar Psychopharmacotheraphy: Caring for the Patient. London: John Wiley \& Sons, Ltd.

Akiskal, H. S. \& Vázquez, G. H. (2006). Una expansión de las fronteras del trastorno bipolar: validación del concepto de espectro. Vertex, 7(69), 340-346.

Akiskal, H., CetkovichBakmas, M., GarcíaBonetto, G., Strejilevich, S., \& Vázquez, G. (2006). Trastornos bipolares. Conceptos clínicos, neurobiológicos y terapéuticos. Buenos Aires: Editorial Médica Panamericana.

American Psychiatric Association (2000). Diagnostic and statistical manual of mental disorders, 4th Edition, Text Revision. Washington, DC: American Psychiatric Association.

American Psychiatric Association (2013). Diagnostic and statistical manual of mental disorders, 5th Edition. Arlington, VA: American Psychiatric Publishing

Angst, J. (1998). The emerging epidemiology of hypomania and bipolar II disorder. Journal of Affective Disorders, 50, 143-151.

Angst, J., Gamma, A., Bowden, C., Azorin, J. M., Perugi, G., Vieta, E., \& Young, A. H. (2012). Diagnostic criteria for bipolarity based on an international sample of 5,635 patients with DSMIV major depressive episodes. European Archives of Psychiatry and Clinical Neuroscience, 262, 311.

Balazs, J., Benazzi, F., Rihmer, Z., Akiskal, K. K., \& Akiskal, H. S. (2006). The close link between suicide attempts and mixed (bipolar) depression: implications for suicide prevention. Journal of Affective Disorders, 91, 133-138.

Baldessarini, R., Tondo, L., Vázquez, G., Undurraga, J., Bolzani, L., Yildiz, A., ... Tohen, M. (2012a). Onsetage versus family history and clinical outcomes in 1665 international bipolarI disorder patients. World Psychiatry, 11(1), 40-46. 
Baldessarini, R., Undurraga, J., Vázquez, G., Tondo, L., Salvatore, P., Ha, K., ... Vieta, E. (2012b). Predominant Recurrence Polarity Among 928 Adult International BipolarI Disorder Patients. Acta Psychiatrica Scandinavica, 125, 293-302.

Disalver, S., Chen, Y., Shoaib, A., \& Swann, A. C. (1999). Phenomenology of mania: Evidence for distinct depressed, dysphoric and euphoric presentations. The American Journal of Psychiatry, 156(3), 426-430.

Dunner, D. L. (2003). Clinical consequences of underrecognized bipolar spectrum disorder. Bipolar Disorders, 5, 456-463.

Geller, B., Zimmerman, B., Williams, M., Bolhofner, K., \& Craney, J. L. (2001). Bipolar disorder at prospective followup of adults who had prepubertal major depressive disorder. The American Journal of Psychiatry, 158, 125-127.

Ghaemi, N. (2003). The Diagnosis of Bipolar Disorder. Posgraduate Course on Bipolar Disorder. Boston: Harvard University.

Goldberg, J. F., Perlis, R. H., Bowden, C. L., Thase, M. E., Miklowitz, D. J., Marangell L. B., ... Sachs, G. S. (2009). Manic symptoms during depressive episodes in 1,380 patients with bipolar disorder: findings from the STEPBD. The American Journal of Psychiatry, 166, 173-181.

Goodwin, F. K. \& Jamison, K. R. (2007). Manicdepressive illness (2da ed). New York: Oxford University Press.

Keitner, G., Solomon, D., \& Ryan, C. (1996). Prodromal and residual symptoms in bipolar I disorder. Comprehensive Psychiatry, 37, 362-367.

Koukoupoulos, A., Sani, G., Koukoupoulos, A. E., Manfredi, G., Pacchiarotti, I., \& Girardi, P. (2007). Melancholia agitata and mixed depression. Acta Psychiatrica Scandinavica Supplementum, 433, 50-57.

Kraepelin, E. (1996). La locura maníacodepresiva. En E. Kraepelin, K. Kalhbaum, \& E. Hecker (Eds.). La locura maníacodepresiva, La catatonía, La hebefrenia (pp. 946). Buenos Aires: Polemos.

Kupfer, D., Frank, E., Grochocinski, V. J., Cluss, P. A., Houck, P. R., \& Stapf, D. A. (2002). Demographic and clinical characteristics of individuals in a bipolar disorder case registry. Journal of Clinical Psychiatry, 63(2), 120-125. 
Kupka, R., Luckenbaugh, D., Post, R., Suppes, T., Altshuler, L. L., Keck, P. E. $\mathrm{Jr}, \ldots$ Nolen W. A. (2005). Comparison of rapidcycling and nonrapidcycling bipolar disorder based on prospective mood ratings in 539 outpatients. The American Journal of Psychiatry, 162(7), 1273-1280.

Lish, J., DimeMeenan, S., \& Whybrow, P. (1994). The National Depressive and ManicDepressive Association (DMDA) survey of bipolar members Journal of Affective Disorders, 31, 28194.

Lolich, M., Vázquez, G., \& Leiderman, E. (2010). Primer episodio psicótico en trastorno bipolar: diferenciación clínica e impacto funcional en una muestra argentina. Vertex, 11(94), 418-427.

Marneros, A., Deister A., \& Rohde A. (1990). The concept of distinct but voluminous groups of bipolar and unipolar diseases. European Archives of Psychiatry and Clinical Neuroscience, 240(2), 77-84.

Merikangas, K., Akiskal, H., Angst J., Greenberg, P. E., Hirschfeld, R. M., Petukhova, M., \& Kessler, R. C. (2007). Lifetime and 12month prevalence of bipolar spectrum disorder in the national comorbidity survey replication. Archives of General Psychiatry, 64(5), 543-552

Nordentoft, M., Mortensen, P., \& Pedersen, C. (2011). Absolute risk of suicide after first hospital contact in mental disorder. Archives of General Psychiatry, 68(10), 1058-1064.

Organización Mundial de la Salud (1992). Clasificación Internacional de Enfermedades y Problemas Relacionados con la Salud, Décima Revisión. Ginebra: OMS.

Perlis, R. (2005). Misdiagnosis of bipolar disorder. The American Journal of Managed Care, 11(9 Suppl), S271-274 .

Perlis, R., Miyahara, S., Marangell, L. B., Wisniewski, S. R., Ostacher, M., DelBello, M. P., ... STEPBD Investigators (2004). Longterm implications of early onset in bipolar disorder: Data from the first 1.000 participants in the Systemic Treatment Enhancement Program for Bipolar Disorder (STEPBD). Biological Psychiatry, 55(9), 875-881.

Rosa, A., Bonnín C., Vázquez G., Reinares, M., Solé, B., Tabarés-Seisdedos, R., ...Vieta, E. (2010). Functional impairment in bipolar II: Is it as disabling as bipolar I? Journal of Affective Disorders, 127(13), 71-76. 
Strejilevich, S. \& Retamal Carrasco, P. (2003). Percepción del impacto y del proceso diagnóstico del trastorno bipolar de personas en tratamiento en centros de Argentina y Chile. Vertex, 14(54), 245-252.

Strejilevich, S., Vázquez G., Garcia Bonetto G., Zaratiegui, R., Vilaprino, J. J., Herbst, L., ... CetkovichBakmas, M. (2010). $2^{\circ}$ Consenso Argentino sobre el Tratamiento de los Trastornos Bipolares. Vertex, 21(Suplemento especial), 255.

Swann, A., Lafer, B., Perugi, G., Frye, M. A., Bauer, M., Bahk, W. M., ... Suppes, T. (2012). Bipolar Mixed States: An International Society for Bipolar Disorders Task Force Report of Symptom Structure, Course of Illness, and Diagnosis. AiA The American Journal of Psychiatry, 170(19), 31-42.

Tondo, L., Vázquez, G., \& Baldessarini, R. (2010). Mania associated with antidepressant treatment: comprehensive metaanalytic review. Acta Psychiatrica Scandinavica, 121(6), 404-414.

Vázquez, G. H., Strejilevich, S., Garcia Bonetto G., CetkovichBakmas, M., Zaratiegui, R., Lagomarsino, A., ... Gutierrez, B. (2005). Consenso Argentino sobre el Tratamiento de los Trastornos Bipolares. Vertex, 16(Suplemento especial), 324.

Vazquez, G., Baldessarini, R., \& Tondo, L. (2014). Cooccurrence of anxiety and bipolar disorders: Clinical and therapeutic overview. Depression and Anxiety, 31, 196-206.

Vázquez, G., Lolich, M., Leiderman, E., Tondo, L., \& Baldessarini, R. (2012). AgeatOnset of 648 Patients with Major Affective Disorders: Clinical and Prognostic Implications. Mind \& Brain, The Journal of Psychiatry, 3(2),17

Vieta, E., \& Suppes, T. (2008). Bipolar II disorder: arguments for and against a distinct diagnostic entity. Bipolar Disorders, 10, 163-178.

Vieta, E., Angst, J., Reed, C., Bertsch, J., Haro, J., \& EMBLEM Advisory Board (2009). Predictors of switching from mania to depression in a large observational study across Europe (EMBLEM). Journal of Affective Disorders, 118, 118-123. 